\title{
Leiomyosarcoma of larynx: A case report
}

\author{
Ashok Gupta · Parvinderjit Singh Kohli
}

\begin{abstract}
Leiomyosarcoma of larynx is a rare tumor. We report a case of 70 year old male who had presented to our department with respiratory distress and stridor. Patient's emergency was managed with tracheostomy with subsequent total laryngectomy and post-op external radiotherapy. One year into follow-up, patient is tumor free.
\end{abstract}

Keywords Leiomyosarcoma - Larynx - Laryngeal Tumor

\section{Introduction}

Leiomyosarcoma is a rare malignant tumor of the head and neck. Approximately, 3 percent of leiomyosarcomas develop in head and neck area [2]. They are rarely found in the larynx, with only 27 cases reported in English literature. We report on the clinical course of a 70 year old patient with a leiomyosarcoma of the larynx.

\section{Case report}

A 70-year-old male presented with stridor to the Department of Otolaryngology, Head and Neck Surgery of Postgraduate Institute of Medical Education and Research, Chandigarh. There was no significant past medical history except that patient was a chronic smoker for last 40 years and had hoarseness of voice for last 3 months. Indirect laryngoscopic examination revealed soft tissue growth completely occluding the glottis. The patient was tracheostomized to manage the emergency. Subsequently, patient was taken up for rigid endoscopic examination and biopsy was taken from the growth. He had no cervical lymphadenopathy. The chest radiograph was normal and the remainder of the examination findings was unremarkable. A contrast enhanced CT scan of the neck revealed a round, soft tissue density mass measuring about $3 \mathrm{~cm}$ at the glottis going into the subglottis upto $2 \mathrm{~cm}$ with no extralaryngeal extension and neck nodes (Fig. 1). Histologic examination of the biopsy showed well differentiated leiomyosarcoma. Immunostains were positive for smooth muscle actin. Total laryngectomy was performed (Fig. 2a). The resection specimen consisted of total larynx with a polypoid tumor arising from both false cords and hanging into the subglottis, measuring $5 \mathrm{x} 4 \mathrm{x}$ $4 \mathrm{~cm}$ (Fig. 2b).

Sectioning of the specimen revealed a yellow to grey solid pattern. Microscopic examination of the resected specimen revealed spindle shaped tumor cells arranged in fascicle and storiform pattern. Tumor cells were pleomorphic with abundant mitotic figures. Immunostains were 

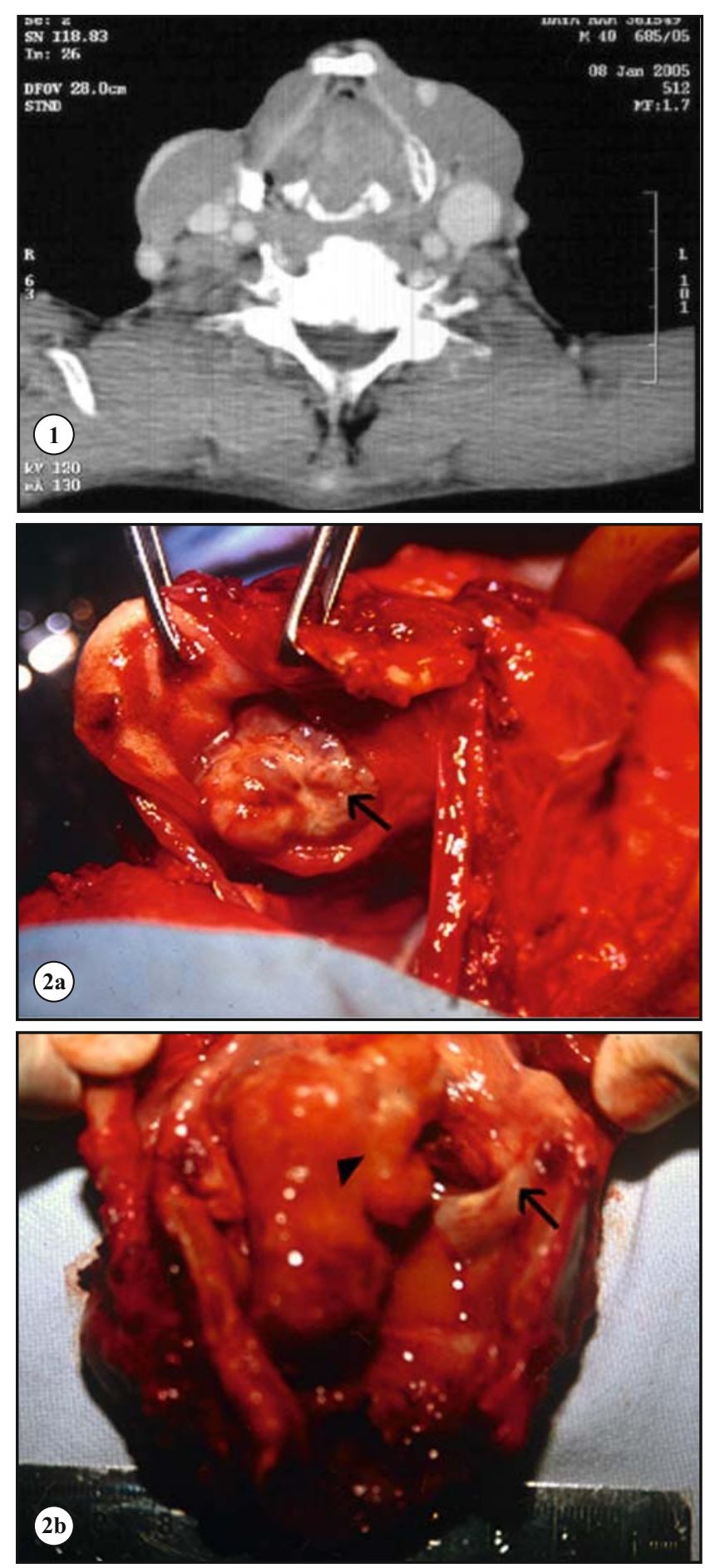

Fig. 1 Contrast enhanced CT scan of the neck showing enhancing soft tissue mass completely filling the subglottis.

Fig. 2 (a) Intraoperative photograph showing (arrow) polypoid tumor completely filling the glottis. (b) Laryngectomy specimen after cutting it open from behind in midline showing a polypoid tumor (arrowhead) starting from left true cord and going into the subglottis without invading the surrounding structures. Free right true cord (arrow). positive for smooth muscle specific actin. Histologically the surgical resection margins were free from tumor. A course of external radiotherapy (50Gy) was given post operatively. One year, on follow up, the patient is free of any recurrences and metastasis and is using Electrolarynx for speech rehabilitation.

\section{Discussion}

The first case of laryngeal leiomyosarcoma was described by Frank in 1941 [4]. This tumor usually occurs in the fifth and sixth decades and has no sexual predilection. Smoking or alcohol abuse does not predispose to this tumor [6, 7]. Symptoms of this tumor (hoarseness, stridor, dysphagia) donot differ from those of other laryngeal malignant tumors [5]. Leiomyosarcoma most commonly originates from glottis followed by supraglottis. Hypopharynx gets involved only as an extension of laryngeal tumor [14].

Three different types of leiomyosarcoma, the 'common' leiomyosarcoma, the vascular leiomyosarcoma and the epitheloid leiomyosarcoma, have been recognized [7]. Histologically, it is characterized by prominent interlacing bundles and fasicles of elongated spindle cells with elongated 'cigar- shaped' blunt-ended nuclei, prominent nucleoli and abundant eosinophilic cytoplasm [5]. The mitotic rate is usually increased and atypical mitotic figures are present. On Immunostaining the leiomyosarcoma is positive for muscle- specific actin and negative for S-100 protein [1]. Desmin is generally negative. The differential diagnosis includes leiomyoma, fibrosarcoma, spindle cell carcinoma, malignant melanoma and inflammatory myofibroblastic tumor [8, 9]. Immunohistochemical, immunocytochemical and ultrastructural investigations are necessary to distinguishing leiomyosarcoma from other spindle- cell tumors.

The potential for metastasis is low [13]. In the cases of well- differentiated leiomyosarcoma, the incidence of cervical lymph node metastasis is low and does not depend upon tumor size. Hematogenous metastases occur infrequently, usually involving the lungs.

Surgery is the treatment of choice [12]. Total laryngectomy is preferred. Post operative external radiotherapy should be given. Radiotherapy alone has no role for this tumor [11]. Chemotherapy has role for palliation in case of distant metastasis.

\section{References}

1. Wadhwa AK, Gallivan H, O'Hara BJ et al (2000) Leiomyosarcoma of the larynx: diagnosis aided by advances in immunohistochemical staining. ENT Journal 79:42-46

2. Chen JM, Novick WH, Logan CA (1991) Leiomyosarcoma of the larynx. Journal of Otolaryngology 20:345-348

3. Gorenstein A, Neel HB, Weiland LH et al (1980) Sarcoma of the larynx. Archives of Otolaryngology 106:8-11

4. Frank DI (1941) Leiomyosarcoma of the larynx. Archives of Otolaryngology 34:493-500 
5. Yoshizaki T, Horikawa T, Nonomura A et al (1999) Leiomyosarcoma of the larynx. Journal of Laryngology Otology 113: 167-169

6. Mcguff HS, Thomas S, Otto RA (1999) Leiomyosarcoma of the larynx. Annals of Otology Rhinology Laryngology 108: 794-796

7. McKiernan DC, Watters GWR (1995) Smooth muscle tumors of the larynx. Journal of Laryngology Otology 109: $77-79$

8. Mindell RS, Calcaterra TC, Ward PH et al (1975) Leiomyosarcoma of the head and neck. Laryngoscope 85: 904-910

9. Rowe-Jones JM, Solomons NB, Rattcliffe NA (1994) Leiomyosarcoma of the larynx. Journal of Laryngology Otology 108:359-362
10. Tewary AK, Pahor AL (1991) Leiomyosarcoma of the larynx: emergency laryngectomy. Journal of Laryngology Otology 105:134-136

11. Cocks H, Quraishi M, Morgan D et al (1999) Leiomyosarcoma of the larynx. Otolaryngology Head and Neck Surgery 121:643-646

12. Kazuyuki K, Masato K, Takeroh I et al (2001) Leiomyosarcoma of the larynx: emergency tracheostomy. Journal of Laryngology Otology 115:570-572

13. Paczano R, Jori J, Tiszlavicz L et al (1999) Leiomyosarcoma of the larynx. Review of the literature and report of two cases. Annals of Otology Rhinology Laryngology 108:677-682

14. Thomas S, McGuff HS, Otto RA (1999) Leiomyosarcoma of the larynx. Case report. Annals of Otology Rhinology Laryngology 108:794-796 